\title{
Why Every Hospital Should (Must) Have an ACE Unit by 2040
}

\author{
Lisa M Walke, MD, MSHA, AGSF1*, S Ryan Greysen, MD, MHS, SFHM²
}

${ }^{1}$ University of Pennsylvania, Department of Medicine, Division of Geriatric Medicine, Philadelphia, Pennsylvania; ${ }^{2}$ Department of Medicine, Division of General Internal Medicine, Section of Hospital Medicine, Perelman School of Medicine at the University of Pennsylvania, Philadelphia, Pennsylvania.

ike the rest of the world, the United States is experiencing an aging boom. The number of adults aged 65 years or older is expected to grow from 49 million in 2016 to 82 million in 2040, indicating an increase of $67 \%$. Even more impressively, the population of individuals aged 85 years or older is expected to increase by $129 \%$ to 14.6 million within this same time period. ${ }^{1}$ Considering that one in five Medicare Fee for Service beneficiaries are hospitalized at least once a year, ${ }^{2}$ hospitals can expect the number of adults over the age of 65 requiring acute care will substantially increase over the next 20 years. These demographic changes have important implications for the overall healthcare costs in the US. Of persons with the highest annual healthcare expenditures, $40 \%$ are 65 years of age or older. ${ }^{3}$ Thus, optimizing the care of hospitalized older adults will remain a critical component in the management of healthcare costs in the next 20 years.

As such, the Acute Care for the Elderly (ACE) unit, an interprofessional model of care that has been shown to provide high-quality care to hospitalized older adults without increasing costs, ${ }^{4}$ will become an increasingly important component of acute care as the older adult population grows. In this edition of the Journal of Hospital Medicine, Brennan et al. ${ }^{5}$ describe a quality improvement initiative in which an interprofessional team that included a geriatric clinician, nurses, pharmacist, and chaplain developed a daily plan of care for ACE unit patients aged 70 years or older. The daily care plan, which focused on symptom management and advance care planning, was the nidus for collaboration between the hospital medicine attending and geriatrics team. Their results demonstrate that ACE unit patients had lower hospital costs and shorter lengths of stay (LOS) as compared with age-matched, usual-care patients despite having higher comorbidity scores. In addition, the greatest benefits were seen among persons in the highest quartile of the comorbidity score.

These results add to the small but consistent body of literature that demonstrates quality and cost benefits to the ACE unit care. Importantly, however, in contrast with the prior ACE unit studies in which persons with moderate risk were the ones to demonstrate the greatest benefits, Brennan et al. ${ }^{5}$ were able to demonstrate the greatest effect for the highest-need,

*Corresponding Author: Lisa M. Walke, MD, MSHA, AGSF; E-mail: lisa.walke@ pennmedicine.upenn.edu; Telephone: 215-898-9401; Twitter: @walke_the_talk

Received: May 20, 2019; Revised: June 26, 2019; Accepted: June 27, 2019

(c) 2019 Society of Hospital Medicine DOI 10.12788/jhm.3278 highest-cost population. Reasons for this impressive effect may be attributed to this intervention's specific emphasis on symptom management and estimated life expectancy. In an era when Medicare and other payers are looking to increase the value proposition in population health-based approaches by reducing high costs while preserving high quality, these findings represent an important example that merits a broader dissemination.

Of course, ACE units are not the only hospital-based programs that have shown to improve outcomes for older adults. The Hospital Elder Life Program (HELP) is an evidence-based delirium prevention intervention that has been shown to not only prevent delirium but also prevent cognitive and functional decline while decreasing hospital LOS, hospital falls, and sitter use. ${ }^{6}$ Moreover, similar to ACE units, HELP has been shown to reduce inhospital patient costs. Geriatrics surgery comanagement programs are another hospital-based intervention that has shown to improve outcomes for older surgical patients. Reductions in LOS, improved mobility, and higher discharge to home have been demonstrated in patients who have undergone spinal surgery? Decreased LOS and lower hospital costs have also been demonstrated among patients with hip fracture undergoing repair. ${ }^{8}$ Programs such as ACE units, HELP, and geriatric surgery comanagement are well aligned with the growing emphasis on value-based healthcare and will be especially needed by hospitals that strive to be high-reliability organizations as the number of adults aged 65 and older continues to grow. To date, few studies have explored the potential synergistic effects (or redundancies) of these programs and how to maximize the impact of these evidence-based interventions across healthcare systems with multiple hospitals that care for older adults from various socioeconomic and cultural backgrounds.

Looking toward the future, the implementation of ACE units and other innovative geriatric programs will equip hospitals to develop into Age-Friendly Health Systems (AFHS). AFHS is an initiative being led by the Institute for Healthcare Improvement, The John A. Hartford Foundation, the American Hospital Association, and the Catholic Health Association of the United States in partnership with several other leading healthcare organizations to provide high-value care to every older adult.9 AFHS provide care focused on the 4M framework-What Matters, Medications, Mobility, and Mentation. The goal is for $20 \%$ of hospitals and medical practices to join the AFHS initiative by 2020; to date, over 70 organizations nationwide have done so. Clearly, to reach this goal, and beyond, a greater collaboration 
between aging-focused interprofessional teams including geriatricians and hospitalists will be essential.

Given the aging demographic and rising healthcare costs, Brennan et al.'s work ${ }^{5}$ suggests that each hospital should have an ACE unit by 2040. Consistently, hospital care delivery has appropriately developed in response to the needs of the patient population served. Intensive care units (ICUs), dialysis units, and emergency rooms are just a few innovations that were adopted by hospitals to provide specialty care to individuals with complex acute illnesses. While technology within the ICU certainly plays a role in the care delivered in that setting, it could be argued that what makes the ICUs most effective is the cohorting of interprofessional expertise. Since the implementation of ICUs, the survival rate for critically ill patients has substantially improved and additional specialty units with an interprofessional team model, eg, cardiac care units, dialysis units, emergency rooms, etc., have followed suit. Specialty units have become a part of the fabric of acute care, so much so that it would be hard to imagine a modern hospital without an ICU, dialysis unit, or emergency room. The same should be true for ACE units. Even hospitals without geriatricians on site can use teleconferencing to successfully implement an ACE unit. ${ }^{10}$ We owe it to our older patients to transform our institutions into AFHS; implementing models of care proven to improve outcomes, such as the ACE unit, is one of the critical first steps.

Disclosures: The authors have no disclosures or financial conflicts of interest. Funding: Dr. Walke was supported by an award from the Health Resources and Services Administration Geriatric Workforce Enhancement Program to the University of Pennsylvania (U1OHP28720).

\section{References}

1. Administration for Community Living. Profile of older adults: 2017. https:// acl.gov/sites/default/files/Aging\%20and\%20Disability\%20in\%20America/2017OlderAmericansProfile.pdf Accessed April 22, 2019.

2. Gorina Y, Pratt LA, Kramarow EA, Elgaddal N. Hospitalization, readmission, and death experience of noninstitutionalized Medicare fee-for-service beneficiaries aged 65 and over. Hyattsville, MD: National Center for Health Statistics. 2015.

3. Agency for Healthcare Research and Quality, Medical Expenditure Panel Survey, Household Component 2015. https://meps.ahrq.gov/data_files/ publications/st506/stat506.shtml Accessed April 1, 2019.

4. Landefeld CS, Palmer RM, Kresevic DM, Fortinsky RH, Kowal J. A randomized trial of care in a hospital medical unit especially designed to improve the functional outcomes of acutely ill older adults. N Engl J Med. 1995;332(20):1338-1344. https://doi.org/10.1056/NEJM199505183322006.

5. Brennan $M$, Knee $A$, Leahy $E$, et al. An acute care for elders $Q$ l program for complex, high cost patients yields savings for the system. J Hosp Med. 2019;14(9):527-533. https://doi.org/10.12788/jhm.3198.

6. Hospital Elder Life Program. https://www.hospitalelderlifeprogram.org/ about/results/ Accessed May 6, 2019.

7. Adogwa $\mathrm{O}$, Elsamadicy $A A$, Vuong VD, et al. Geriatric comanagement reduces perioperative complications and shortens duration of hospital stay after lumbar spine surgery: a prospective single-institution experience. J Neurosurg Spine. 2017;27(6):670-675. https://doi.org/10.3171/2017.5.SPINE17199.

8. Della Rocca GJ, Moylan KC, Crist BD, Volgas DA, Stannard JP, Mehr DR. Comanagement of geriatric patients with hip fracutues: a retrospective, controlled, cohort study. Geriatr Orthop Surg \& Rehab.2013;4(1):10-15. https:// doi.org/10.1177/2151458513495238.

9. Institute for Healthcare Improvement. http://www.ihi.org/Engage/Initiatives/ Age-Friendly-Health-Systems/Pages/default.aspx. Accessed May 6, 2019

10. Malone ML, Vollbrecht M, Stephenson J, Burke L, Pagel P, Goodwin JS. Acute Care for Elders (ACE) tracker and e-geriatrician: methods to disseminate ACE concepts to hospitals with no geriatricians on staff. J Am Geriatr Soc. 2010;58(1):161-167. https://doi.org/10.1111/j.1532-5415.2009.02624.x. 